\title{
Untreated surgical conditions in Malawi: A randomised cross-sectional nationwide household survey
}

\author{
Carlos Varela ${ }^{1,2,3}$, Sven Young ${ }^{1,2,4}$, Reinou Groen ${ }^{6,7}$, Leonard Banza ${ }^{1,2,3}$, \\ Nyengo C. Mkandawire ${ }^{8}$, Asgaut Viste ${ }^{3,5}$ \\ 1. Kamuzu Central Hospital, Lilongwe, Malawi \\ 2. Lilongwe Campus, College of Medicine, University of Malawi, Lilongwe, Malawi \\ 3. Institute of Clinical Sciences (K1) and Centre for International Health, University of Bergen, Bergen, Norway \\ 4. Department of Orthopaedic Surgery, Haukeland University Hospital, Bergen, Norway \\ 5. Department of Acute and Gastrointestinal Surgery, Haukeland University Hospital, Bergen, Norway \\ 6. Department of Gynecology and Obstetrics, Johns Hopkins Hospital, Baltimore, Maryland, USA \\ 7. Department of Obstetrics and Gynaecology, Alaska Native Medical Center, Anchorage, Alaska, USA \\ 8. College of Medicine, University of Malawi, Blantyre, Malawi
}

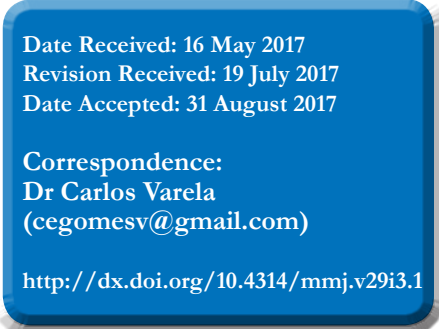

\section{Background}

Noncommunicable diseases, such as surgical conditions have received little attention from public health planners in low income countries (LIC) like Malawi. Though increasingly recognised as a growing global health problem, the burden of surgical pathologies and access to surgical care has not been adequately identified in many LIC. Information on the spectrum and burden of surgical disease in Malawi is important to uncover the unmet need for surgery and for planning of the National Health Service.

Methods

This was a multistage random cluster sampling national survey. Households were selected from clusters using probability proportional to size method. 1448 households and 2909 interviewees were analysed. The Surgeons Overseas Assessment of Surgical need (SOSAS) tool was used to collect data. This electronic tablet based questionnaire tool included general information and a dual personalised head to toe inquiry on surgical conditions. The general information included number of household members, and inquired on any death within the past twelve months, and if any of the deaths in the family had a suspected surgical condition leading to that death. Data was collected by specially trained third year medical students.

Results

Out of 1480 selected households, 1448 (98\%) agreed to participate, with 2909 interviewed individuals included in the study. The median household size was 6 individuals (range $1-47$ ). Median age of interviewed persons was 35 years (range $0.25-104$ years). 1027 out of $2909(35 \%)$ of the interviewed people reported to be living with a condition requiring surgical consultation or intervention, whereas 146 of $616(24 \%)$ of the total deaths reported to have occurred in the preceding 12 months were reported to have died from a surgically related condition. Most individuals did not seek health care due to lack of funds for transportation to the health facility. Only $3.1 \%$ of those that reported a surgical condition had surgical intervention.

Conclusions

There is a large unmet need for surgical care in Malawi. A third of the population is living with a condition needing surgical consultation or intervention, and a quarter of all deaths are potentially avoidable with surgery. Urgent scale up of surgical services and training are needed to reduce this huge gap in public health planning in the country.

\section{Introduction}

Noncommunicable diseases, including many surgical conditions, have been neglected for a long time in public health planning in low income countries. ${ }^{1}$ Though surgical diseases and injuries are increasingly being recognized as a growing global health problem, ${ }^{1}$ the burden of these conditions has not been adequately described in most of Sub Saharan Africa. Some low income countries, including some African nations, have however carried out national surveys to describe the situation in their country, such as Nepal, Sierra Leone, Rwanda and Uganda. ${ }^{1-}$

Many surgical conditions can lead to avoidable death if there is delay in seeking health care. Conditions such as incarcerated or strangulated hernias, bowel obstruction, appendicitis, peritonitis, congenital anomalies and some tumours can be treated surgically if identified and referred to a surgical facility early enough. Unfortunately, many of these conditions are neglected in low income countries, especially in the rural communities. Traumatic injuries, if neglected, can also cause significant delay in recovery, permanent disability or death. This can have a profound negative effect on a family's social and economic situation and even the economic development of the whole country. ${ }^{17}$

The lack of data on the burden of surgical diseases and injuries in Malawi is a threat to effective health sector planning, and can perpetuate the current under funding of surgical services. The aim of this study was to estimate the burden of surgical conditions in Malawi.

\section{Methods}

\section{Setting}

Malawi is a low income country in South Eastern Africa with a population of 16.8 Million, ${ }^{7}$ and one of the lowest GDP per capita in the world at USD 294 in $2016 .{ }^{13}$ Only $77 \%$ of public service posts for health personnel are filled, with approximately 40 surgeons in total, of which $20 \%$ are in the private sector. With the lowest density of surgeons in the World, Malawi has 0.24 surgeons per $100,000 .{ }^{16}$ It is estimated that the number of facilities with adequate staff 


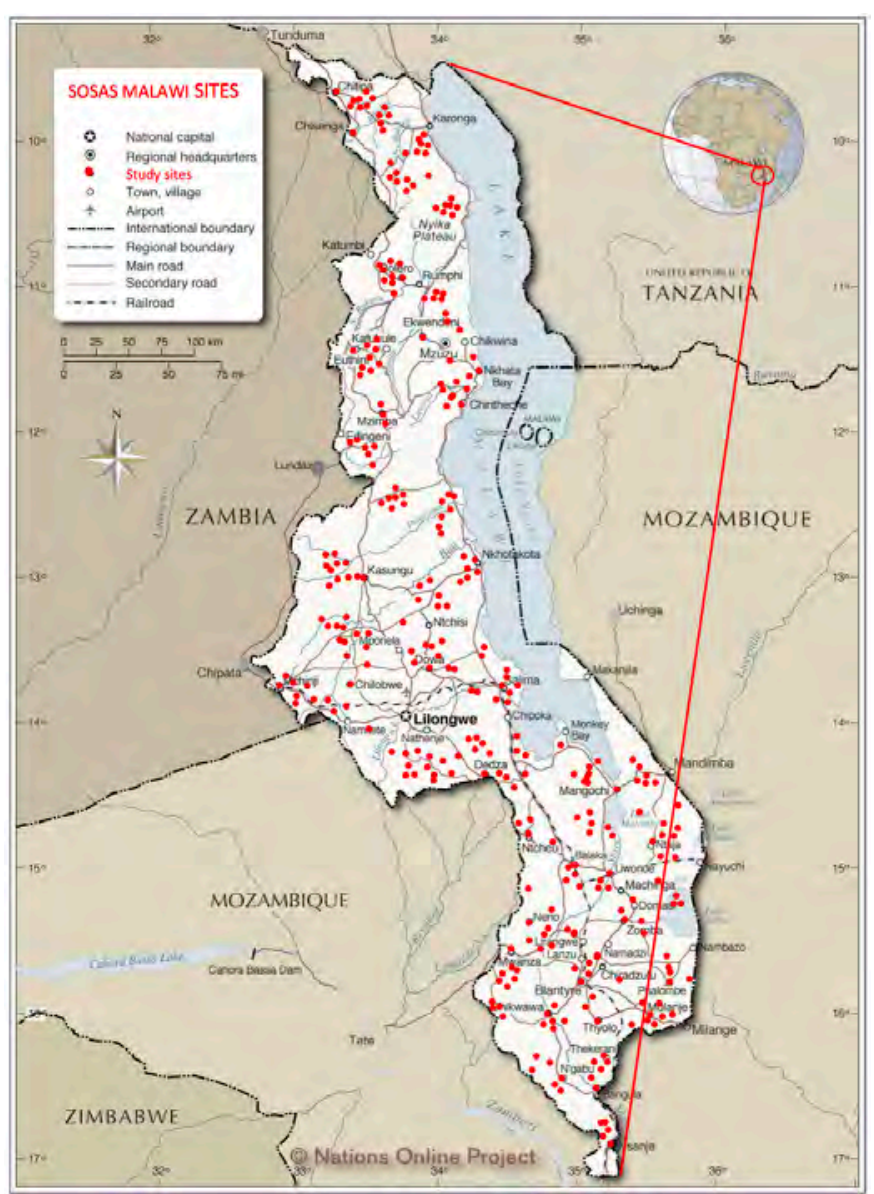

Figure 1: Malawi map showing the areas from which the settlements were selected

to implement the WHO defined Essential health package (EHP) is $9.2 \%$ of the total number of health facilities. Malawi is divided into North, Central and Southern regions, with the majority $(80 \%, 12.5$ million) of the population living in rural areas. ${ }^{14}$ The Central and Southern regions are the most densely populated with 6.4 and 6.8 million respectively and the Northern region the least densely populated region. ${ }^{14}$

There are a total of 28 districts in the country, with a total number of 48,233 registered s (villages and towns). The majority of these settlements are rural communities dependent on subsistence farming and small scale businesses. 27 districts are on the mainland, while 1 district is an island on Lake Malawi.

\section{Study design}

This was a cross-sectional multistage national survey. The sample size was estimated to be 1497 households from a pilot study that was carried out in rural areas of the capital city, Lilongwe which estimated the combined untreated surgical conditions to be at $25 \%$. The pilot study preceded the main survey by 5 months to validate the use of the SOSAS tool. The sample size for the individuals was estimated at 2994 individuals $(95 \% \mathrm{CI})$ with a design effect of 1.5 , at a $25 \%$ prevalence of unmet surgical need.

The National statistics office provided the data on settlement areas from the Malawi Census Board for 2008 national census records with 48,233 recorded settlements. All the 48,233 settlements were randomised through computer generated random numbers using an Excel application, selecting 55 settlements as potential enumeration areas from each district on the mainland for this survey. From the selected 55 settlements from each of the districts, 16 per district were selected using probability proportion to size for sampling. Two or four households were randomly selected in each settlement depending on size. Two households were selected in a settlement with less than 10 households, while 4 households were selected in larger settlements, with more than 10 households. The household randomisation was based on a floor bottle spin and picking the fifth house in the direction of spin. Two household members were selected and interviewed per household, by first interviewing the senior member present, and then selecting another member at random using random numbers based on number of members in the household. When a member of the household under the age of 6 years was randomly selected, the actual information was given by their guardian. The total number of included households was 1448, with a total of 2909 people interviewed.

\section{Survey instrument}

The Surgeons Overseas Assessment of Surgical need tool (SOSAS) was used to collect data. ${ }^{1,2}$ The authors received permission to use the SOSAS tool for no cost. This is a questionnaire-based tool with three components. The first component outlines the general household information and demographics. The second part is a personalised interview, with specific questions on symptoms and findings from head to toe, of the same person who gave the general information. The third component involved a random second household member who was also interviewed on any current surgical conditions from head to toe. The questionnaire was installed on a tablet computer (iPad 2, Apple Inc., Cupertino, CA, USA), using FileMaker Pro 12.0v3 (FileMaker Inc., Santa Clara, CA, USA) software. This electronic tablet was also used to capture visible surgical pathologies for certainty. The questionnaire was translated into 2 local languages; Chichewa and Tumbuka prior to installation on the tablet. The collected data was exported from the tablets into an Excel (Microsoft 2010) database at the end of each day in the field for the entire period of data collection.

\section{Data collection}

Data collection was done by medical students who had just finished their 3rd year and underwent tool training for 5 days as a refresher of the training for the pilot study. They all underwent ten days of training on how to use the questionnaire and computer tablet prior to the pilot study. There were in total 32 trained data collectors, with 16 interviewers in the field at a time. The period for data collection was from 1st July to 30th August 2016, and was spilt into 2 phases. The first phase involved half of the data collectors covering all identified enumeration sites in the northern part of the Central Region and the whole of Northern Region. While the second phase involved coverage of the rest of Central region and the Southern Region of the country. In some of the enumeration areas people belonged to smaller population groups with unique languages or dialects. In this case translators were hired to secure good communication. Each data collector was covering 1 or 2 settlements per day to interview 2 or 4 household depending on the size of the settlement; therefore $32-64$ households were interviewed each day in total. This was confirmed by checking the number of entries at the end of the day for each of the individual data collectors. Random checking of the data entry forms was done at the end of each day to reassure quality of the data. 
Table 1: General household information and demographics

$(\mathrm{N}=1448)$

$\%$

\section{Household data}

\section{Location}

Rural

91.2

Urban

Median household size (n)

6 (range 1-47)

Respondents

\section{Sex distribution}

$$
\text { Male }
$$

Females

Median age (years)

35 (range 0.25-104)

\section{Individual data}

Respondents

98

\section{Education level}

None

Primary

Secondary

Tertiary

Graduate degree

\section{Occupation}

Unemployed

House builder

Domestic helper

Farmer

Own business

Government employee

NGO employee

3.5

\section{Statistical analysis}

Data analysis was done using STATA 13.1 (StataCorp LP, College Station, TX, USA) and SPSS version 24. Analyses were done for univariate statistics and multivariate logistic regression model was also done to predict the untreated surgical conditions (Present surgical condition yes or no). Chi square tests were used for univariate associations for contingency tables. Normal distribution for data and skewness for data were checked by t-test and Mann-Whitney $\mathrm{U}$ test.

\section{Ethical considerations}

The study was approved by the Malawi College of Medicine Research Ethics Committee (approval: P03/15/1696), and Norwegian Regional Research ethics committee (approval: 2016/1392/REK Vest). Consent and assent forms were translated into the local languages and used to seek informed consent prior to conducting the interviews.

Table 2: Reported surgical conditions requiring consultation or surgery

\begin{tabular}{lcc}
\hline Condition & $\mathrm{n}$ & $\%$ \\
\hline Wound (injury-related) & 177 & 6.0 \\
Wound (not injury-related) & 116 & 4.0 \\
Burn & 45 & 1.5 \\
Solid mass & 258 & 8.8 \\
Soft mass/reducible & 140 & 4.8 \\
Congenital deformity & 124 & 4.3 \\
Acquired deformity & 107 & 3.7 \\
Abdominal distension/mass & 42 & 1.4 \\
Urological complaints & 14 & 0.5 \\
Rectal bleed & 16 & 0.5 \\
\hline Total & $\mathbf{1 0 3 9}$ & $\mathbf{3 5 . 5}$
\end{tabular}

Table 3: Anatomical locations of conditions requiring surgery

\begin{tabular}{lcc}
\hline Anatomical region & n & \% \\
\hline Face/head/neck & 240 & 8 \\
Chest & 81 & 3 \\
Abdomen & 261 & 9 \\
Back & 63 & 2 \\
Groin/genitalia & 81 & 3 \\
Extremities & 301 & 10 \\
\hline Total & $\mathbf{1 0 2 7}$ & $\mathbf{3 5}$
\end{tabular}

\section{Results}

The enumeration areas incorporated all the districts except for Likoma, which is an island where access is only by chartered plane or the once weekly ferry. Figure 1 shows the location of the areas from which the settlements were selected. This was provided by the national census board and was used for randomisation for the survey. The majority of households were rural (91\%) while $9 \%$ were urban. The median number of household occupancy was 6 (Range 1 47) individuals, with a median age of 35 years (Range 0.25 - 104), (Table 1).

Out of the total targeted 1480 households, 1471 households consented and were included, representing $99 \%$ of the respondent households and 1448 were analysed data. $2909(98 \%)$ out of 2951 individuals consented and were analysed (Table 1). Data from 23 households were excluded due to entry missing values. Nine households refused to give consent for involvement, while 23 individuals refused to give consent, and 42 individuals had missing values on sex and age.

There were 1039 (35.3\%; 95\% CI 33.8 - 38.6) reported surgical conditions in total (Table 2). In 140 individuals there was more than one complaint existing at the time of the interviews which accounted for the multiple presentation of conditions.

1027 out of 2909 analysed individuals (35\%: $95 \%$ CI $32.6-$ 37.4) confirmed having a mass, congenital birth defect, burn deformity or contracture, or other surgical condition at the time of interview, and indicated that they required a surgical consultation or intervention. Extrapolated to the population size of Malawi this gives an estimated 5.5 million people living with a surgical condition in Malawi at the time of this study. 
'Table 4: Comparison of the situation in select African countries and Nepal

\begin{tabular}{llccc}
\hline Country & Population & GDP/capita (US\$) & Untreated surgical conditions (\%) & $\begin{array}{c}\text { Mortality from untreated } \\
\text { surgical conditions (\%) }\end{array}$ \\
\hline Malawi & $16.8 \mathrm{~m}$ & 294 & 35 & 24 \\
Sierra Leone & $6.3 \mathrm{~m}$ & 808 & 25 & 25 \\
Uganda & $34.6 \mathrm{~m}$ & 726 & 11 & 34 \\
Rwanda & $12.4 \mathrm{~m}$ & 722 & 6 & 33 \\
Nepal & $28.5 \mathrm{~m}$ & 689 & 10 & 23
\end{tabular}

By pathology, solid masses were predominant, followed by injury wounds and also congenital defects (Table 2). By anatomic location, the predominant conditions were in the extremities, i.e. conditions of feet, legs, hands and arms followed by abdominal conditions, face, head and neck lesions (Table 3).

597 households reported at least one death in the family in the previous year, with overall 616 reported deaths in the past 12 months. $146(24 \%)$ out of 616 reported deaths were due to a surgically related condition (Table 2). Conditions assumed to be surgical in nature were; traumatic and nontraumatic wounds, abdominal distension, neck and other body swellings; Chest mass, Breast mass, groin mass, limb mass and other visible surface masses.

For multivariate analysis, the parameters used for comparison were, sex, current illness and illness in the past 12 months. Females were more likely to report or seek consultation than males (O.R 2.32 95\% CI 2.1 - 2.6). Those that had recovered from the illness in the past 12 months were less likely to report an illness present at the current moment as having a sick health status (O.R 1.67 95\% CI 1.21-1.93).

The crude death rate due to surgical disease was estimated at 67 per 1000 population per year (total household occupancy of 8644, 616 dead household members).

\section{Discussion}

This study uncovered a huge unmet burden of surgical disease and mortality in Malawi. The findings are comparable to those from other African countries, but, to our knowledge, at $35 \%$, this is the highest reported prevalence of surgical disease in an African Country to date. The unmet need for surgical services in Malawi is evident. Many people are living with a condition needing surgical consultation or surgery. This study also found that $24 \%$ of all deaths reported over the last year were due to a surgically treatable condition.

The SOSAS tool has been used in other Low income countries to estimate the burden of surgical disease and a comparison of the main findings from these countries can be found in Table 4. Our findings in Malawi were similar to those in Sierra Leone, where $25 \%$ of the population was found to have a surgical condition and $25 \%$ of mortality was estimated to be due to a surgical condition. ${ }^{1,2}$ Malawi is a low income country with a GDP per capita of 294 and a population close to 17 million. ${ }^{13,14}$ Although the Population of Malawi is three times that of Sierra Leone, with a GDP of almost a third of that of Sierra Leone, the estimated unmet need of the burden of surgical diseases is comparable in these two countries. These relatively similar findings of surgical burden and mortality from a surgical related cause when compared to other countries could be explained by similarities of the general healthcare set up and lack of adequate surgical care at the rural community level (Table 4). The crude death rate is also similar to that of Sierra Leone SOSAS survey, reflecting the inadequacy of health services in these countries. Mortality due to related untreated surgical disease is equally high in other countries that have conducted a SOSAS survey. In Rwanda, Uganda and Nepal, mortality due to a surgical disease was estimated to be even higher than in our study (Table 4).

It has been estimated that in 48 African countries, 288 million people are living with a surgically treatable condition, and that 5.6 million deaths could be averted by surgical intervention annually. ${ }^{9}$ These extrapolated figures from three previous SOSAS studies demonstrate the clear need of surgical care scale up in the African region as a whole. It is high time surgical conditions become a main stream part of the global health discussion.

One of the main limitations of this survey is recall of events, especially deaths, in the household. If the family member died some months prior, the family member could not clearly disclose the cause and circumstances surrounding the death either due to recall effects or that culturally they are not comfortable discussing it. This could contribute to recall and information bias on data collection although the information was confirmed from the deceased person's health passport book if available, or from the household head.

Another limitation could also be the reporting and description of the surgical condition. This was based on inquiry of the condition, and not examining the persons with their complaint. This would have required extra time, and more medically qualified enumerators, to conduct the physical examination on them. But realistically for a resource limited country like Malawi, it would not be feasible to get 30 medically qualified clinicians out for a 60 days' national survey and deprive the health facilities from service provision. Hence it was not possible within the timeframe and budget of this study. Some of the conditions, though interpreted as surgical, were not confirmed as such, and could have been wrongly classified. However, the opposite is also possible, and we do not think this is a major bias. Some congenital conditions, like cardiac septal defects, are not easily diagnosed without clinical examination. Some conditions like umbilical hernias or other body swellings are sometimes not considered as a problem, especially if some other family member has had a similar condition and it never caused any problem. So this required a lot of inquiry without actually examining the condition, otherwise it could be presented as a normal finding.

There are many other causes of mortality from communicable diseases, like malaria, tuberculosis and immune suppression 
related illnesses. Some of these conditions are not in isolation, but coexist with some surgical illness. Not all will require a surgical procedure for intervention, but even just a surgical consultation. This could either raise the incidence or vice versa if the diagnoses is missed.

Some surgical conditions are believed to be inherited and not harmful, like hernias and hydroceles. This could be one of the reasons the communities believe there is no need for them to seek health care from the facilities. Other communities believe the condition they have cannot be treated or even associate the condition with witchcraft. This may have been a contributing factor to not seeking health care for some.

Misunderstandings due to language could be another limitation. Malawi has 2 official languages; English and Chichewa, but many areas also have their own local languages. Though interpreters were engaged, some descriptions of medical conditions needed thorough explanation to understand what they were referring to. The questionnaire was only translated into 2 local languages, Chichewa for the central and the southern region, and Tumbuka, mostly spoken in the northern part of Malawi.

Some traumatic conditions are seasonal in Malawi, such as the high frequency of falls from trees in the mango season and collapsing houses during the rains, and therefore could not be captured fully in this study conducted in the cold, dry ("winter") season. In winter, burns are more common due to people using fire in their houses to keep warm.

In the rural areas of Malawi, transportation costs are high for people with little cash income, and the roads often become muddy and un-serviceable during the rainy season. Many people in the rural communities either present late, or do not present at all to the health facility due to transport problems, lack of funds, or not knowing the need to visit the health facility. ${ }^{15}$ Others get discouraged because the local health centre will not have the necessary equipment and drugs. ${ }^{10}$ They would rather wait till they have sourced enough funds to enable them to visit a secondary level health facility, a district hospital, or even a tertiary centre, a central hospital, of which only 4 exist for the entire population of 16 million. These district hospitals and central hospitals are usually far from these rural communities, and also faced with inadequate supplies and inadequate human resource to offer the services. ${ }^{10-12}$ These economic, social and geographical factors can all lead to delay in contacting the health system and explain why so many people are living with untreated surgical conditions.

Untreated surgical conditions are widespread in Malawian rural communities and there is a large potential to prevent the complications of such conditions if presentation to a health facility that can provide good surgical service is done early enough. With the knowledge of this, it could be sensible to promote community awareness of certain common pathologies that can be surgically treated. Alignment of funds for programs addressing surgical conditions by training health care workers to recognise, treat or refer surgical cases to the appropriate level of care in a timely manner could have a large impact. Surgical team visits could also be organised to rural areas by specialists from the central hospitals to make travel less of a barrier for the population in order to get health care. Strengthening of the surgical capacity in Malawi is urgently needed. With the severe resource limitations in Malawi, surgical service scale up can be most efficiently reached by sufficient support of the existing surgical training programmes in the main cities. Once a critical mass of surgeons has been trained, they can expand outreach services to district hospitals and rural health centres with the goal of also producing surgeons for the districts in the future.

\section{Conclusions}

This study has uncovered a huge unmet burden of surgical disease and mortality in Malawi. One-third of the population is living with a surgically correctable condition, and one quarter of all reported deaths is potentially avoidable with surgery. Urgent scale up of surgical services and training programmes are needed to reduce this huge gap in public health service in the country.

\section{Acknowledgements}

\section{Funding}

Tablet computers were obtained through support from the NORHED project for support of surgical training and research in Malawi (https://www.norad.no/en/ front/funding/norhed/projects/capacity-building-inpostgraduate-surgical-training-and-research-in-malawi/) and were donated to the surgical department at $\mathrm{KCH}$ for use in further research. Costs for data collectors, fuel, meals and accommodation while travelling to the different areas throughout the country during data collection were covered by the same programme.

\section{Competing interests}

All authors declare that they have no competing interests related to this work.

\section{References}

1. Groen RS, Samai M, Stewart KA, Cassidy LD, Kamara TB, Yambasu SE, et al. Untreated surgical conditions in Sierra Leone: A cluster randomized, cross-sectional, countrywide survey. Lancet 2012, Aug 13; 380 (9847): 1082-7.

2. Groen RS, Samai M, PetrozeRT, Kamara TB, Yambasu SE, CallandJF, et al. Pilot testing of a population-based surgical survey tool in Sierra Leone. World J Surg 2012, Apr; 36 (4): 771-4.

3. Tu. M Tran, Anthony T. Fuller, Elissa K. Butler, Frederick Makumbi, Sammuel Luboga, Christine Muhumuza, Vincent F. Ssennono, Geffrey G. Chipman, Moses Galukande, Michael. M. Haglund. Burden of Surgical conditions in Uganda. Ann Surg 08 Sep 2016;266(2):389-399.

4. Elissa K. Butler1, Tu M. Tran, Anthony T. Fuller, Alexa Brammell, Joao Ricardo Vissoci, Luciano de Andrade, Fredrick Makumbi, Samuel Luboga, Christine Muhumuza, Vincent F. Ssennono, Jeffrey G. Chipman, Moses Galukande, Michael M. Haglund, Emily R. Smith. Quantifying the pediatric surgical need in Uganda: results of a nationwide cross-sectional, household survey. Pediatr Surg Int. 2016 Nov;32(11):1075-1085.

5. Stewart KA, Groen RS, Kamara TB, Farahzad MM, Samai M, Cassidy LD, et al. Traumatic injuries in developing countries: Report from a nationwide cross-sectional survey of Sierra Leone. JAMASurg 2013, Jan 16: 1-7.

6. van Loenhout JA, Delbiso TD, Gupta S, Amatya K, Kushner AL, Gil Cuesta J, Guha-Sapir D. Barriers to surgical care in Nepal. BMC Health Serv Res. 2017 Jan 23;17(1):72.

7. Richard Record and Abdu Mohiddin. An economic perspective on Malawi's medical "brain drain" Globalization and Health 2006, 2:12 doi:10.1186/1744-8603-2-12

8. PetrozeRT, Groen RS, Niyonkuru F, Mallory M, Ntaganda E, Joharifard S, et al. Estimating operative disease prevalence in a lowincome country: Results of a nationwide population survey in Rwanda. Surgery 2012, Dec 17. 
9. Shailvi Gupta, Reinou S Groen, Patrick Kyamanywa, Emmanuel A Ameh, Mohamed Labib, Damian L Clarke, Peter Donkor, Miliard Derbew, Rachid Sani, Thaim B Kamara, Sunil Shrestha, Benedict C Nwomeh, Sherry M Wren, Raymond R Price, Adam L Kushner. Surgical care needs of low-resource populations: an estimate of the prevalence of surgically treatable conditions and avoidable deaths in 48 countries. Online publish. The Lancet.com April 27, 2015

10. Moses Galukande, Johan von Schreeb Essential Surgery at the District Hospital: A Retrospective Descriptive Analysis in Three African Countries. PLoS Medicine |plosmedici 2010 | Volume 7 | Issue 3 | e1000243

11. Weiser TG,Regenbogen SE, Thompson KD, Haynes AB, Lipsitz SR et al. (2008) An estimation of global volume of surgery: a modelling strategy based on available data. Lancet 372: 139-144

12. Margaret E. Kruk, Andreas Wladis Human Resource and Funding Constraints for Essential Surgery in District Hospitals in Africa: A Retrospective Cross-Sectional Survey PLoS Medicine | plosmedic 2010 | Volume 7 | Issue 3 | e1000242

\section{IMF, World Economic Outlook database, 2016}

14. Malawi population data sheet 2012

15. Young S, Banza LN, Hallan G, Beniyasi F, Manda KG, Munthali BS, et al. Complications after intramedullary nailing of femoral fractures in a low-income country. Acta Orthop. 2013; 84 (5): 460-7

16. Eric O’Flynn, Judith Andrew, Avril Hutch, Caitrin Kelly, Pankaj Jani, Ignatius Kakande, Miliard Derbew, Sean Tierney, Nyengo Mkandawire, Krikor Erzingatsian. The Specialist Surgeon Workforce in East, Central and Southern Africa: A Situation Analysis. World J Surg (2016) 40:2620-2627 DOI 10.1007/s00268-016-3601-3

17. Haug L, Wazakili M, Young S, Van den Bergh G. Longstanding pain and social strain: patients' and health care providers' experiences with fracture management by skeletal traction; a qualitative study from Malawi. PubMed.gov 2016 Jul 20: 1-8 